\title{
Superior vena cava syndrome due to pacemaker leads: An Indian report with a review of management practice
}

\author{
Shekhar Kunal ${ }^{1}$, Vijay Pathak ${ }^{1}$, Pooja Pathak ${ }^{2}$ \\ ${ }^{1}$ Department of Cardiology, Sawai Man Singh Medical College, Jaipur; ${ }^{2}$ Lady Health Officer, NIMS Medical College, \\ Jaipur, India
}

\begin{abstract}
Superior vena cava (SVC) syndrome due to pacemaker leads is a distinct diagnostic rarity. It is diagnosed clinically based on constellation of signs and symptoms supported by imaging evidence of SVC obstruction. A 70-year-old male, an ex-smoker, presented with facial and upper limb swelling along with exertional dyspnea. He had undergone transvenous dual chamber rate modulated (DDDR) pacemaker implantation four years back for symptomatic sinus node dysfunction. Imaging demonstrated a hypodense-filling defect in the SVC causing 70-80\% luminal narrowing along with presence of collaterals. Patient was managed on anticoagulants and planned for SVC stenting with epicardial pacing which failed. Patient refused to undergo any further treatment and was subsequently lost to follow-up. To the best of our knowledge, this is the first detailed description of SVC syndrome due to pacemaker leads from India, which was managed medically.
\end{abstract}

Correspondence: Vijay Pathak, Department of Cardiology, Sawai Man Singh Medical College, Jaipur, Rajasthan 302 004, India.

Mobile: +91.98290 .52082 .

E-mail: vijalps2000@yahoo.com

Conflict of interest: The Authors declare no conflict of interest.

Key words: Superior vena cava syndrome; pacemaker leads; thrombosis; anticoagulants; balloon angioplasty.

Contributions: VP, study concept, responsible for the genuineness of the data, guarantor of the paper; SK, VP, clinical data collection; PP, assistance in review of the literature. All the authors drafted the manuscript, and have read and approved the final manuscript.

Received for publication: 4 July 2018.

Accepted for publication: 29 January 2019.

(C) Copyright S. Kunal et al., 2019

Licensee PAGEPress, Italy

Monaldi Archives for Chest Disease 2019; 89:977

doi: 10.4081/monaldi.2019.977

This article is distributed under the terms of the Creative Commons Attribution Noncommercial License (by-nc 4.0) which permits any noncommercial use, distribution, and reproduction in any medium, provided the original author(s) and source are credited.

\section{Introduction}

Superior vena cava (SVC), the largest systemic vein of the mediastinum, is formed by the confluence of right and left brachiocephalic veins and drains upper part of the body into right atrium. Azygous vein, a major tributary of the SVC, forms a greater part of the drainage in cases of SVC obstruction [1]. SVC syndrome, an entity first described by William Hunter in 1757 [2], refers to the obstruction of venous flow in the superior vena cava. The diagnosis of SVC syndrome is clinical based on presence of characteristic signs and symptoms with imaging and other investigations being supportive [1]. In the pre-antibiotic era, SVC syndrome usually occurred due to infectious diseases such as syphilitic aortic aneurysm and tuberculosis. Nowadays, malignancy especially adenocarcinoma of the lung, breast and mediastinal masses are the leading cause [1]. In the last two decades, thrombotic occlusion of SVC due to presence of central venous catheters as well as pacemaker leads is frequently being encountered [3].

SVC syndrome due to the presence of pacemaker leads is a diagnostic rarity with just a single case being reported in the literature from the Indian subcontinent [4]. In addition, a lack of definite treatment strategy for this entity further leads to inadequate and inappropriate management. We report the case of a 70-yearold male who presented with SVC syndrome due to pacemaker leads and was subsequently managed on anticoagulants and antithrombotic agents.

\section{Case Report}

A 70-year-old male was referred for evaluation of episodic dizziness and swelling of face and arms for the past 20 days. He had complained about multiple episodes of light-headedness along with headache, which increased on bending forwards. In addition, swelling over the face in form of facial puffiness, which had progressively involved both the arms and upper part of the chest wall. His clinical course was also marked by development of exertional dyspnea over the past 20 days, usually while climbing stairs. A lack of clinical response to oral medications as well as inhalers had prompted the referral. He had been an ex-smoker with 18 pack-years of smoking. His previous medical history comprised implantation of transvenous dual chamber rate modulated (DDDR) pacemaker (Medtronic RELIA ${ }^{\circledR}$ RED01) four years back for symptomatic sinus node dysfunction. There was no history of fever, cough, haemoptysis, hoarseness of voice, dysphagia and weight loss. General physical examination revealed an elderly male in no acute respiratory distress with marked swelling over 
the face, both upper limbs as well as upper part of the chest. In addition, a positive Pemberton's sign and distended veins over the anterior chest wall with a flow from above downwards were noted. Respiratory and cardiovascular system examination were within normal limits. A provisional diagnosis of SVC syndrome was made and the patient further investigated to determine its aetiology.

Routine blood investigations including liver and renal functions were normal. Programming of the DDDR device reported normal functioning of the pacemaker with the atrial and ventricular pacing percentages being $98 \%$ each (APVP mode) suggesting the patient to be pacemaker dependent. Chest radiograph postero-anterior view (Figure 1) revealed widening of the superior mediastinum with pulse generator and pacemaker lead in situ. Doppler ultrasound of the neck was suggestive of dilated bilateral jugular venous system with stagnant flow on the right side suggestive of superior vena caval obstruction. Contrast enhanced computed tomography (CECT) of chest and neck revealed a hypodense filling defect in the SVC causing 70-80\% luminal narrowing suggestive of a thrombus (Figures 2 and 3). Multiple collateral network was seen along with a prominent calibre of the azygous vein. Subcutaneous tissue oedema was noted in bilateral chest wall as well as retropharyngeal region and myofascial planes of the neck. Atrial and ventricular leads were also visualised without any evidence of lead fracture or displacement. A diagnosis of SVC syndrome due to pacemaker leads was made. The patient was apprised of the treatment options including the need for extraction of the pacemaker leads and epicardial leads placement followed by stenting of the diseased portion of SVC. Low-molecular weight heparin (LMWH) along with warfarin was initiated as the initial treatment modality. There was subsidence in the facial and chest wall oedema following anticoagulant therapy. Epicardial pacing was attempted but it failed and the patient was planned for transfemoral placement of the leads along with extrac-

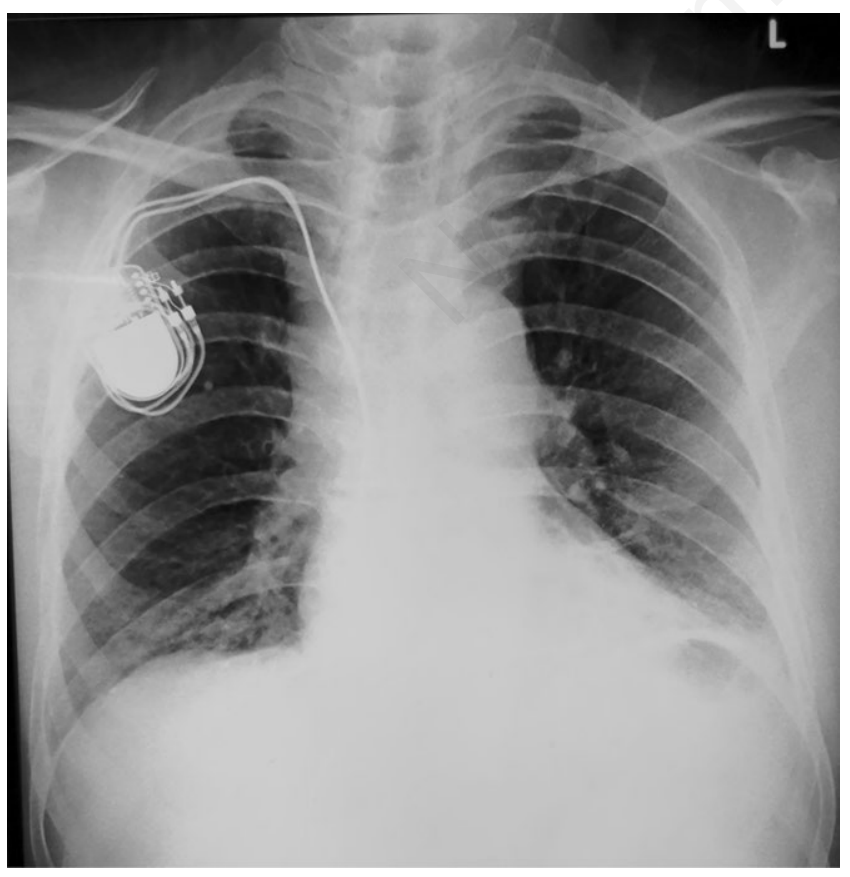

Figure 1. Chest radiograph postero-anterior view showing widening of the superior mediastinum along with presence of pacemaker leads and pulse generator. tion of the pre-existing pacemaker leads followed by stenting of SVC. The patient however, refused to undergo transfemoral lead placement and was continued on injection LMWH along with oral anti-coagulants for a week. He had marked improvement in his symptoms and was subsequently discharged on oral anti-coagulants. He was then lost to further follow-up.

\section{Discussion}

The occurrence of thromboembolic complications following transvenous pacemaker implantation has been reported to occur in $0.6-3.5 \%$ with SVC syndrome developing in less than $0.1 \%$ of patients [5]. Wertheimer et al. [6] had first described pacemaker lead induced SVC syndrome in 1973. Since then, various reports and case series have highlighted this rare but a serious complication carrying a significant morbidity and mortality.

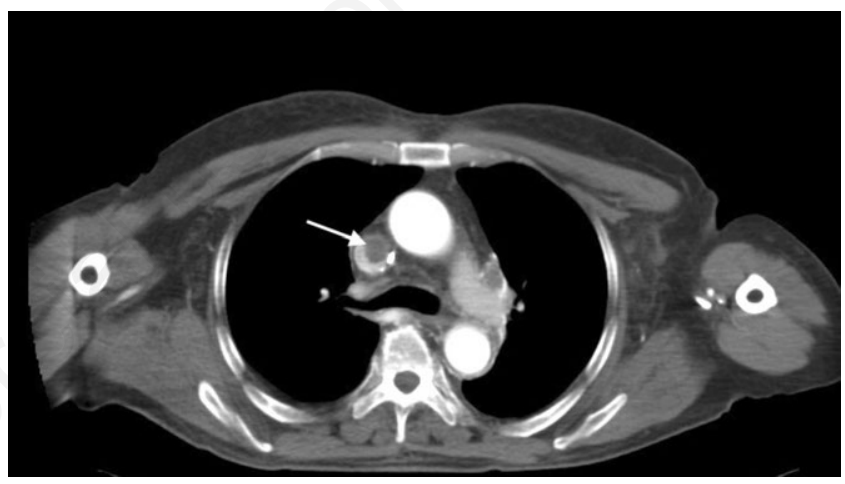

Figure 2. Contrast enhanced computed tomography (axial section) showing presence of a hypodense filling defect in the superior vena cava (white arrow) causing marked luminal narrowing along with presence of pacemaker lead.

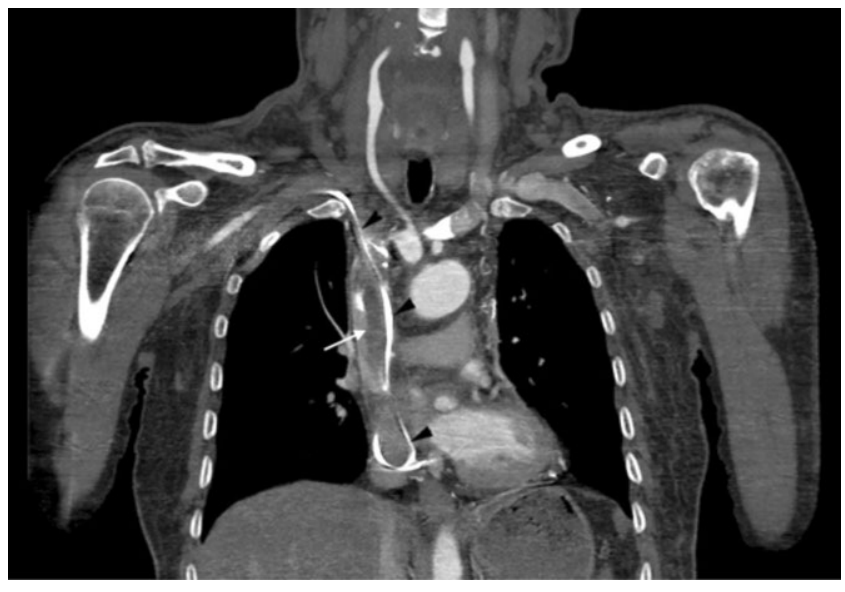

Figure 3. Contrast enhanced computed tomography (coronal section) showing presence of a linear hypodense thrombus in the superior vena cava causing $\mathbf{7 0 - 8 0 \%}$ luminal narrowing (white arrow) with the presence of pacemaker leads (black arrow heads). 
This clinical entity develops as a result of either thrombosis or stenosis of the SVC, which usually occurs close to its junction with right atrium. Repeated episodes of trauma from the pacemaker lead results in disruption of the venous endothelial lining. This culminates in vessel wall inflammation followed by fibrin deposition, thrombus formation and ultimately scarring and stenosis [7]. Sometimes partial recanalization of the thrombus may also lead to fibrotic narrowing of SVC. Factors increasing the risk of thrombus formation include: i) presence of multiple endocardial leads; ii) lead infection; iii) temporary pacemaker wires prior to permanent device implantation; iv) presence of severed leads; v) hormonal therapy; and vi) prior history of venous thrombosis $[7,8]$.

Clinical presentation varies from being asymptomatic in majority of patients to a more severe form of the disease with neurological obtundation and airway obstruction being the initial manifestation. This depends on acuteness of obstruction as well as the degree of collateralization of blood flow. The major collateral channels include i) azygos-hemiazygos system, ii) internal mammary vessels, iii) lateral thoracic thoracoepigastric vessels, iv) vertebral vessels and small mediastinal veins [1]. The most common presenting symptoms include neck, facial and upper extremity swelling, dyspnoea, cough, hoarseness of voice, dysphagia and facial plethora. Neurological symptoms suggestive of cerebral oedema such as headache, confusion, dizziness and obtundation are rare. The findings on clinical examination include the presence of oedema on face, neck and upper extremities along with distended veins on neck and anterior chest wall [1]. In addition, certain clinical findings such as cervical or axillary lymphadenopathy can often be a diagnostic clue. Diagnosis is usually established based on clinical suspicion followed by confirmation on imaging including conventional contrast/CT venography. Contrast venography is considered to be the gold standard for diagnosing venous obstruction and localization of stenotic lesion and is often done prior to an interventional procedure [1]. It does carry an advantage over CT venography in terms of i) demonstration of degree of obstruction, ii) visualisation of opacified collaterals and iii) the extent of thrombi in peripheral vasculature [9]. CT venography along with CT chest has a sensitivity of $96 \%$ and specificity of $92 \%$ for the diagnosis of SVC syndrome [9]. It not only detects the venous thrombosis but also helps to rule out other pulmonary aetiologies especially malignant neoplasms. Duplex scanning too helps in detection of thrombotic occlusion based on demonstration of a lack of compressibility and decreased flow as was seen in our case.

Though SVC syndrome due to pacemaker lead has been reported worldwide, a search of PubMed, IndMed and other databases revealed that it has been previously reported only once from India [4]. The authors reported a 47-year-old female who underwent transvenous pacemaker implantation for sick sinus syndrome. Subsequently, she developed SVC syndrome 10 years post pacemaker implantation, which was managed surgically. Our case highlights the medical management of SVC syndrome with its early detection and subsequent conservative management using anticoagulants.

Management options in patients with pacemaker lead induced SVC syndrome seem to be haphazard with no clear-cut guidelines having been laid down. As compared to SVC syndrome caused by malignancies, treatment of benign causes such as pacemaker lead induced often takes a longer time with recurrences. Treatment depends upon the timing of presentation, resources and the technical expertise available. Anticoagulation and thrombolysis are useful in patients with acute presentation and have moderate efficacy [10]. Long-term anticoagulation, as was done in our case, is usually warranted in pacemaker lead induced venous thrombosis. Thrombolysis with streptokinase and recombinant tissue plasmino- gen activator has been successful in thrombotic SVC obstruction however, carries a risk of partial clot lysis and fragmentation with subsequent pulmonary embolism $[8,10]$. Another valid strategy is percutaneous transluminal balloon venoplasty in patients with either thrombosis (early presentation) or venous stenosis (delayed presentation). The results are not promising due to high rates of restenosis. Balloon angioplasty and stent implantation does have better outcome especially in cases with fibrotic narrowing of SVC. However, stent implantation is fraught with dangers of lead entrapment leading to pacing failure $[5,10]$. This can be mitigated by lead extraction followed by stenting and then lead reimplantation, a technique with considerable success in short and medium term follow up [10]. Life-long anticoagulant therapy is warranted post definite endovascular therapy owing to the high risk of re-occlusion.

A lot of debate exists regarding the removal of the pacemaker leads as it poses a great technical challenge. These leads may get incorporated into the vessel wall or the cardiac chambers hence removal can often lead to complications and vascular injury. Lead extraction alone may cause development of large intimal flaps and thrombosis, leading to restenosis. Patients with SVC obstruction refractory to endovascular treatment can undergo surgical interventions such as a SVC bypass using a spiral saphenous vein conduit or the reconstruction of the SVC using a pericardial patch followed by the epicardial pacing [4]. In a retrospective review of the treatment practices from 74 published studies comprising 104 patients, it was seen that the main treatment modality was anticoagulation in $29 / 104$ patients. This was followed by stenting in $25 / 104$, surgery in $23 / 104$, venoplasty in $16 / 104$ and thrombolysis in $11 / 104$ patients [8]. This review also highlighted that anticoagulation, thrombolysis, and venoplasty alone had higher recurrence rate whilst those managed with surgery and stenting had better outcomes [8].

\section{Conclusions}

This report highlights one of the rare complications of pacemaker implantation as well as focuses on the need for standardization of management practices. One must be cautious enough to look for thromboembolic complications during follow-up in patients with permanent pacemakers as early identification and treatment of this clinical entity prevents unnecessary morbidity and mortality. In developing countries such as India with a substantial burden of elderly population and better medical care, the frequency of permanent pacemaker/Implantable Cardioverter Defibrillator implantation has increased. Thus, there is a need to educate the physicians regarding this interesting albeit rare complication.

\section{References}

1. Wilson LD, Detterbeck FC, Yahalom J. Clinical practice. Superior vena cava syndrome with malignant causes. N Engl J Med 2007;356:1862-9.

2. William $\mathrm{H}$. The history of the aorta, with some remarks on aneurysms in general. Med Observ Inq 1757;1:323-57.

3. Rice TW, Rodriguez RM, Light RW. The superior vena cava syndrome: clinical characteristics and evolving etiology. Medicine (Baltimore) 2006;85:37-42.

4. Madkaiker AN, Krishna N, Jose R, et al. Superior vena cava syndrome caused by pacemaker leads. Ann Thorac Surg 2016;101:2358-61. 
5. Barakat K, Robinson NM, Spurrell RA. Transvenous pacing lead-induced thrombosis: a series of cases with a review of the literature. Cardiology 2000;93:142-8.

6. Wertheimer M, Hughes RK, Castle CH. Superior vena cava syndrome. Complication of permanent transvenous endocardial cardiac pacing. JAMA 1973;224:1172-3.

7. De Costa SS, Scalabrini NA, Costa R, et al. Incidence and risk factors of upper extremity deep vein lesions after permanent transvenous pacemaker implant. A 6-month follow-up prospective study. Pacing Clin Electrophysiol 2002;25:1301-6.
8. Riley R, Peterson S, Ferguson J, et al. Managing superior vena cava syndrome as a complication of pacemaker implantation: A pooled analysis of clinical practice. Pacing Clin Electrophysiol 2010;33:420-5.

9. Straka C, Ying J, Kong FM, et al. Review of evolving etiologies, implications and treatment strategies for the superior vena cava syndrome. Springerplus 2016;5:229.

10. Fu HX, Huang XM, Zhong L, et al. Outcome and management of pacemaker-induced superior vena cava syndrome. Pacing Clin Electrophysiol 2014;37:1470-6. 